\title{
DIAGNOSTIC ACCURACY OF MRI IN CHARACTERIZING THE OVARIAN MASSES INDETERMINATE ON COLOR DOPPLER ULTRASOUND.
}

1. DMRD, FCPS (Radiology) Associate Professor

Department of Radiology

Allied Hospital /

Faisalabad Medical University,

Faisalabad.

2. FCPS (Radiology)

Assistant Professor

Department of Radiology

Allied Hospital /

Faisalabad Medical University,

Faisalabad.

3. FCPS (Radiology)

Assistant Professor

Department of Radiology

Madina Teaching Hospital,

Faisalabad.

4. MBBS, DMRD

Assistant Professor

Department of Radiology

Govt. General Hospital, G.M. Abad

Faisalabad Medical University,

Faisalabad.

Correspondence Address:

Dr. Syed Anjum Mehdi

Department of Radiology

Allied Hospital,

Punjab Medical College, Faisalabad.

dranjumehdi@gmail.com

Article received on:

08/04/2019

Accepted for publication:

05/11/2019

\begin{abstract}
Syed Anjum Mehdi ${ }^{1}$, Hassan Bukhari ${ }^{2}$, Shama Aslam ${ }^{3}$, M. Sohail Anjum Noor ${ }^{4}$
\end{abstract}
ABSTRACT... Objectives: Aim of my study is to determine the diagnostic accuracy of MRI in characterization of indeterminate ovarian masses on Doppler ultrasound (US) of pelvis while keeping histopathology as gold standard. Study Design: Cross sectional (validation) study. Setting: Radiology Department of Allied Hospital Faisalabad. Period: From January 2015 to December 2016. Material and Methods: Approval was taken from institutional ethical committee, a total of 91 patients were enrolled in this study. All the patients found to have indeterminate ovarian mass on sonography and Doppler US were examined with MR imaging performed on a 1.5-T Philips MR imaging unit in the radiology department Allied Hospital Faisalabad. The interval between Doppler US and MRI pelvis was about 7 to 10 days. Histopathology was taken as gold standard for final diagnosis and in determining the diagnostic accuracy of MRI. Results: All women having a mean age of 42 years (range, 15-85 years) who found to have indeterminate ovarian masses on Doppler US were included in the study. There were $71.4 \%$ benign and $28.6 \%$ malignant cases found on MRI while on histopathology $77 \%$ masses were benign and $23 \%$ malignant. The sensitivity and specificity of MRI in determining adnexal masses in our study is $100 \%$ \& $93.86 \%$ Conclusion: MRI is ideal imaging modality for characterization of indeterminate ovarian masses. Magnetic resonance imaging is found to be quiet helpful in characterization of ovarian masses where sonography and Doppler US are not helpful.

Key words: Adnexal/ Ovarian Masses, Doppler Ultrasound, Magnetic Resonance Imaging.

Article Citation: Mehdi SA, Bukhari H, Aslam S, Noor MSA. Diagnostic accuracy of MRI in characterizing the ovarian masses indeterminate on color Doppler ultrasound. Professional Med J 2019; 26(12):2250-2254.

DOI: 10.29309/TPMJ/2019.26.12.3532

\section{INTRODUCTION}

Among the gynecological cancers, Ovarian cancer is the leading cause of death, old age women with advanced ovarian cancer have the best survival with proper therapeutic options. ${ }^{1}$

Ovarian masses create challenging situation for preop diagnosis that is whether the lesion is benign or malignant. Sensitivity of transvaginal ultrasound is very high for ovarian masses but its specificity is lower to rule out malignant lesions. ${ }^{2}$ When Doppler US is used to characterize benign or malignant ovarian lesions, it has improved the specificity and positive predictive value for detection of malignant lesions. ${ }^{3}$ Spectral analysis in form of resistive index (RI) and pulsality index (PI) of Doppler waveform has been widely used in detecting the status of blood flow in lesion characterization. ${ }^{4}$

Due to advent of radiological investigations mortality rate due to ovarian cancer has declined to $0.7 \% .^{5}$ It is found that few of lesions having pure benign characters as pure simple cysts were found malignant on histopathology especially in patients having risk factor of previous breast cancer or ovarian cancer. ${ }^{6}$

Approximately $5 \%-10 \%$ of U.S. women with suspected adnexal mass undergo surgery, but in only $0.38 \%-18 \%$ of these patients mass was diagnosed to be malignant. ${ }^{7}$

The limitation with sonography and Doppler ultrasound is that of experience of sonographer. ${ }^{1}$ The role of CA 125 and screening ultrasound are 
still debatable. For ovarian cancer, screening is currently not routinely advised, but in high-risk groups i.e. females with a family history of cancer, screening is recommended. Even in these high risk groups a large number of benign masses are found on surgery. ${ }^{8}$ Important step after finding an indeterminate ovarian mass is whether to go for direct surgery or further investigation i.e. spelvic $\mathrm{MRI}$ which is very important in characterization of ovarian masses ${ }^{9}$ and it helps in preventing unnecessary surgeries and in anticipating ovarian carcinoma before operation. ${ }^{10}$

Recently, treatment of benign lesions is mostly with laparoscopy while unnecessary laparotomy which is extensive surgery and with certain complications can be avoided. ${ }^{11}$ It was found that MRI was having an accuracy of $95 \%$ for malignant ovarian masses, with a PPV of 0.92 and NPV of $0.98 .{ }^{12}$ which were significantly higher than Doppler USG.

\section{MATERIAL \& METHODS}

Thestudy was conducted in Radiology Department of Allied Hospital Faisalabad. Duration of study was 2years from January 2015 to December 2016. Permission was taken from hospital ethical committee. Patients are collected from OPD of Radiology and gynecology department of Allied Hospital Faisalabad. Written Informed consent was taken from patient after explaining objective $\&$ procedure of the study. An inclusion criterion was set as female patients having indeterminate ovarian masses on TVS. Exclusion criteria were all patients having already diagnosed malignancy, pacemakers or claustrophobic patients. On Doppler resistive index (RI) and pulsality index (PI) $s$ values were measured and a cut off of 0.55 and 1.0 respectively were taken, lower than of these values were considered malignant lesions. For $\mathrm{MRI}$ of pelvis certain advises were given to patients regarding safety of $\mathrm{MRI}$ and Patients were also advised to fast for $3-4 \mathrm{hrs}$, not to urinate for $4-5 \mathrm{hrs}$ before the examination to allow a moderately filled bladder. All the patients were examined with MR imaging performed on a 1.5-T Philips MR imaging unit. A sense abdomen coil was used in all patients. $5 \mathrm{~mm}$ thick slice were taken of the following sequences: Axial T1-weighted (W),
Axial, Sagittal, coronal T2W sequences in every patient while Axial T2W fat suppressed images without contrast and axial, coronal and sagittal T1W contrast-enhanced images were obtained after IV injection of gadolinium $0.1 \mathrm{mmol} / \mathrm{kg}$ body weight as per requirement of the patient.

The patients with suspicious of malignancy were operated by senior gynecologist while true cut biopsy/ FNAC was taken from benign looking lesions and histopathology was done from histopathology lab PMC/Allied Hospital Faisalabad and were reported by senior histopathologist.

\section{RESULTS}

Statistical data obtained were i.e. on MRI $71 \%$ masses were benign and $28 \%$ were malignant (table1) however on histopathology $77 \%$ were benign and $23 \%$ were malignant (table2), so sensitivity of $\mathrm{MRI}$ for malignant masses was $100 \%$ and specificity 93.8\%.(table3). Excellent agreement was found between MRI and Histopathological diagnosis for determining the origin of adnexal masses ( $\mathrm{k}=0.92)$.

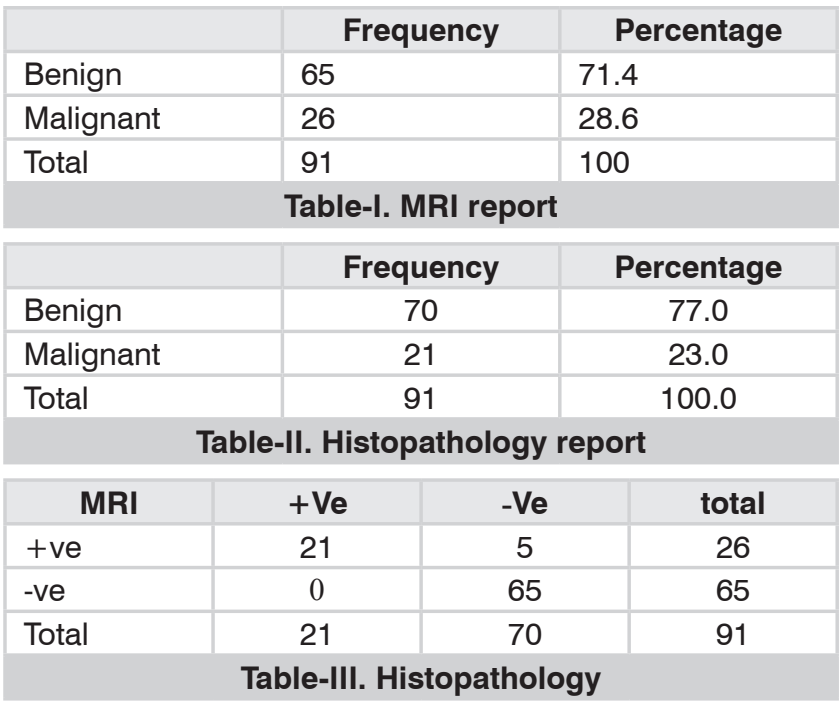

Value 95\% confidence interval

Sensitivity $\quad 100 \%(83.89 \%$ to $100.00 \%)$

Specificity $\quad 93.86 \%(84.11 \%$ to $97.64 \%)$

PPV $\quad 80.77 \%$ (64.35\% to $90.72 \%)$

NPV $\quad 100 \%(91.97,100)$

Diagnostic Accuracy 95.08\% (86.51, 98.31) 


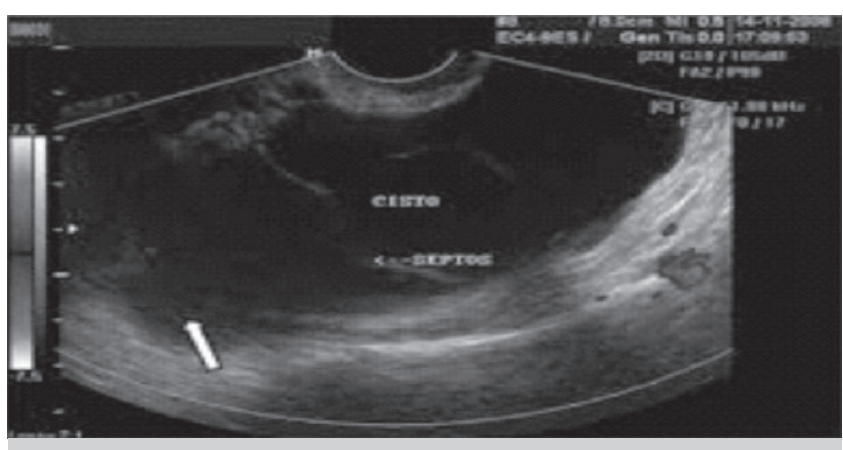

Case I: On Doppler USG a complex cystic lesion with think internal septa and solid component at periphery shown.

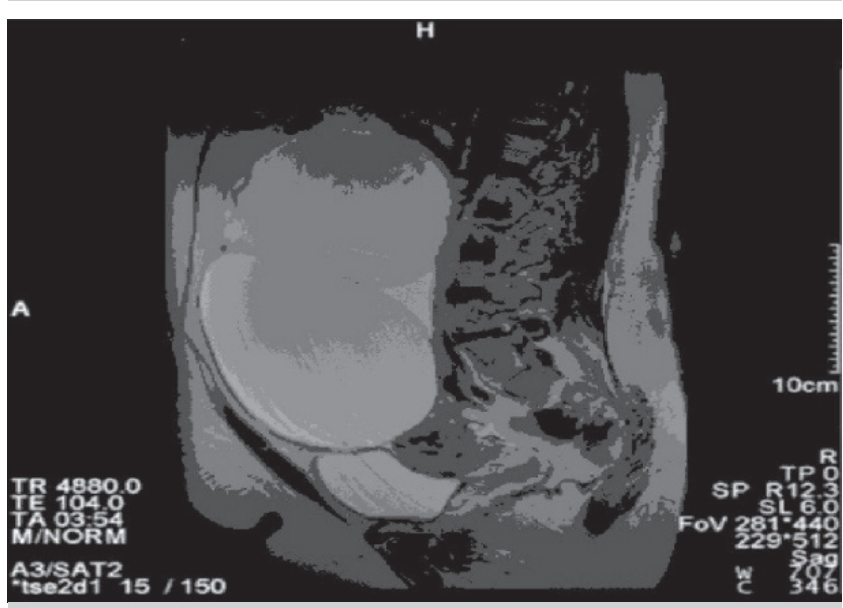

Case II: MRI pelvis of same pt. showing findings consistent with Doppler scan suggesting malignant ovarian lesion which was confirmed on histopathology

\section{DISCUSSION}

Incidence of pelvis mass in life time of female is $20 \%{ }^{13}$ and if it diagnosed at early stage, survival is $90 \%$.

For evaluation of ovarian masses Transabdominal/ Transvaginal sonography (TVS) is the first line of diagnostic modality. But the specificity of TVS for the definitive diagnosis of ovarian malignancy has limited value. ${ }^{14}$

According to study by Shyamala who found that Doppler US has sensitivity, specificity positive predictive value and negative predictive values of $96.29 \%, 84.04 \%, 78.79 \%$, and $97.37 \%$ respectively in predicting malignant lesions when $\mathrm{RI}$ of 0.55 is used as cut off value. ${ }^{4}$

In equivocal findings of Doppler US the modality of choice is MRI. In a study by Tukeva et al, he compared TVS/ Doppler US findings with MRI

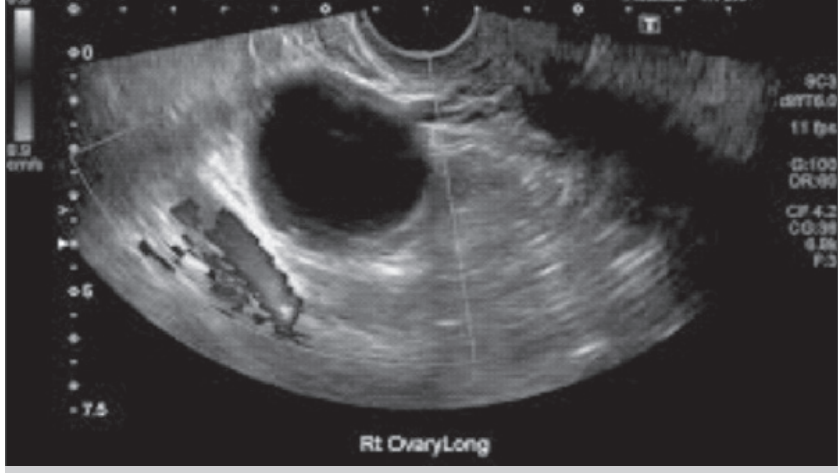

Case I: On Doppler scan, a complex cystic lesion seen in right ovary having thick wall along its base with no significant vascularity within.

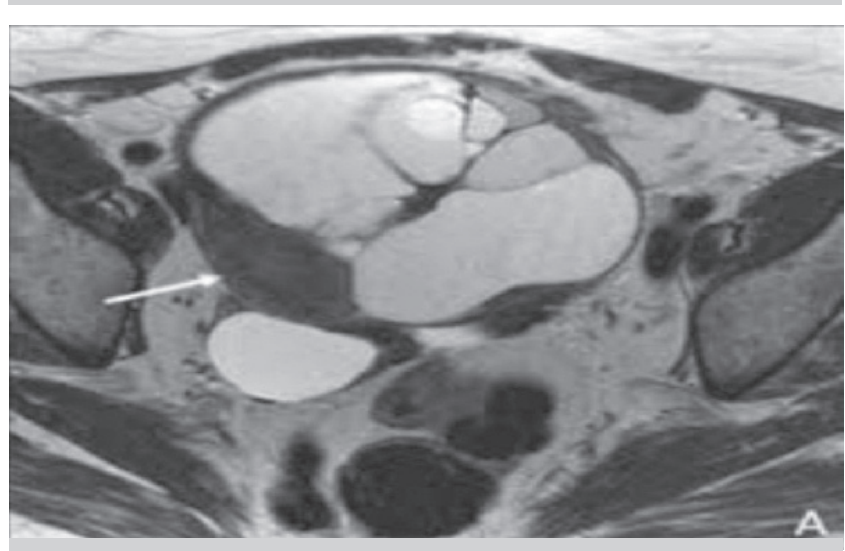

Case II: MRI pelvis of same patient showing complex cystic lesion which is having thick solid component at its base which shows significant enhancement (not shown) so diagnosis of ovarian carcinoma was made and confirmed on histopathology

pelvis and found MRI to be more accurate than TVS in the diagnosis of PID. ${ }^{15}$

In one study by Khurana ${ }^{16}$, who found that using cut off of 1.0 for PI value, sensitivity, specificity, positive predictive value and negative predictive values were $86.67 \%, 68.42 \%, 68.42 \%$ and $86.67 \%$ respectively in characterizing malignant ovarian lesions.

The results of study shows that MRI is investigation of choice for indeterminate ovarian masses, so unnecessary surgeries may be prevented. According to my study, $72.1 \%$ adnexal masses were benign on $\mathrm{MRI}$ and $27.9 \%$ were malignant while on histopathology $77 \%$ masses were benign and $23 \%$ malignant. The sensitivity and specificity of MRI in determining adnexal masses in our study was $100 \%$ \& $93.6 \%$ respectively which are consistent with the study of Adusumilli S. Et al. ${ }^{17}$ Who found that when used for further 
evaluation of an indeterminate adnexal mass in a prospective series, MRI pelvis showed sensitivity and specificity of $100 \%$ and $94 \%$, respectively, in diagnosis of malignancy.

The results of our study are also comparable to that of Kinkel et al. ${ }^{18}$ who found that no doubt MRI can be helpful in cancer detection, the main contribution of MRI in characterization of adnexal masses is its specificity.

Women with suspected adnexal masses, for identifying malignant lesions both Doppler ultrasound and $\mathrm{MRI}$ were highly sensitive (ultrasound 100\%, MRI 96.6\%), but significantly greater specificity of MRI was found (ultrasound $39.5 \%$, MRI 83.7\%). Therefore, women who have a low risk of malignancy on clinical findings but on ultrasound indeterminate lesions were found are the ones most likely to benefit from MRI. ${ }^{19}$

In one study it was found that patients who were diagnosed at later for which 5-year survival rates are on average $16 \%$ to $27 \% .{ }^{20}$

According to Hricaket al. ${ }^{21}$ overall accuracy for differentiating benign from malignant adnexal tumors MRI pelvis has been shown to have overall accuracy of $91-93 \%$.

Sonography (transvaginal and transabdominal) and Doppler US are sensitive methods for detecting ovarian cancer. They showed the sensitivity sonography was $87.5 \% .^{22}$

According to lyer Doppler US was shown to have a sensitivity of $84 \%$ and a specificity of $82 \%$ in characterization of malignant lesions. ${ }^{23}$

The soft tissue characterization of MRI is ideal to reveal normal ovaries separately and also to determine the characteristic morphologic features of a mass. ${ }^{24}$

\section{CONCLUSION}

Results of our study show that the MRI pelvis is choice of investigation in determining indeterminate ovarian masses on Doppler ultrasound. The sensitivity and specificity of
MRI in our patients is comparable to the results found in different studies conducted before which is significantly higher than Doppler scans. MRI is considered as a choice of investigation for characterization of indeterminate ovarian lesions. MRI is good option not only to prevent unnecessary surgeries but also to decrease the burden on hospital and doctors as well.

Copyright $(05$ Nov, 2019.

\section{REFERENCES}

1. Jenny J. MD, Ovarian cancer treatment and survival trends among women older than 65 years of age in the United States, 1995-2008. Obstetrics \& Gynecology. 2016; 127: 81-89.

2. Noam S, Ron M, Assessment of adnexal masses using ultrasound: A practical review. Int J Womens Health. 2014; 6: 857-863.

3. Liu Z, Yang F, Zhang Y, Conventional, doppler and contrast-enhanced ultrasonography in differential diagnosis of ovarian masses, Cell Physiol Biochem 2016; 39:2398-2408.

4. Jothy $M$, Anju $p$, Sonomorphology and colour flow Doppler studies in differentiating between benign and malignant ovarian masses, 2017; 6(2):626.

5. Mona S,Steben M, Watson M, "Evolution of cervical cancer screening and prevention in United States and Canada: implications for public health practitioners and clinicians." Preventive Medicine. 2013; 57: 426-33.

6. Valentin L, Ameye L, Franchi D, et al. Risk of malignancy in unilocular cysts: A study of 1148 adnexal masses classified as unilocular cysts at transvaginal ultrasound and review of the literature. Ultrasound Obstet Gynecol. 2013; 41:80-89.

7. Demir RH, Marchand GJ, Adnexal masses suspected to be benign treated with laparoscopy. JSLS. 2012; 16: 71-84.

8. Jacobs IJ, Menon U, Maharaj AG, Ovarian cancer screening and mortality in the UK Collaborative Trial of Ovarian Cancer Screening (UKCTOCS): A randomised controlled trial. Lancet 2016; 387: 94556.

9. Pavlik EJ, Ueland FR, Miller RW, et al. Frequency and disposition of ovarian abnormalities followed with serial transvaginal ultrasonography. ObstetGynecol 2013; 122:210.

10. Forstner $R$, Meissnitzer $M$. Update on imaging of ovarian cancer. 2016; 4:31. 
11. Pulcinelli F.M, Schimberni M, Marci R. Laparoscopic versus laparotomic surgery for adnexal masses: role in elderly. World Journal of Surgical Oncology. 2016; 14:105.

12. Guerra A, Cunha TM, Félix A. Magnetic resonance evaluation of adnexal masses. Acta Radiol. 2008; 49:700-9

13. R. G. Moore and R. C. Bast Jr., "How do you distinguish a malignant pelvic mass from a benign pelvic mass? Imaging, biomarkers, or none of the above," Journal of Clinical Oncology, 2007; 25: 4159-61.

14. Fleischer $A$, Lyshchik $A$, Jones $H$, Diagnostic parameters to differentiate benign from malignant ovarian masses with contrast-enhanced transvaginal sonography, J Ultrasound Med 2009; 28:1273-1280.

15. Tukeva TA, Aronen HJ, Karjalainen PT, Molander $P$, Paavonen $T$, Paavonen J. MR imaging in pelvic inflammatory disease: Comparison with laparoscopy and US. Radiology. 1999 Jan. 210(1):209-16.

16. Khurana1 I, Satia N. Preoperative evaluation of ovarian masses with color Doppler and its correlation with pathological finding, 2016; 5(7): 2089.

17. Adusumilli S, Hussain HK, Caoili EM, Weadock WJ, Murray JP, Johnson TD, et al. MRI of sonographically indeterminate adnexal masses. AJR Am J Roentgenol. 2006; 187:732-40.

18. Kinkel K, Lu Y, Mehdizade A, Pelte MF, Hricak $H$. Indeterminate ovarian mass at US: incremental value of second imaging test for characterization metaanalysis and Bayesian analysis. Radiology. 2005; 236:85-94.
19. Sohaib SA, Mills TD, Sahdev A, Webb A, Vantrappen $\mathrm{PO}$, Jacobs IJ, et al. The role of magnetic resonance imaging and ultrasound in patients with adnexal masses. ClinRadiol. 2005; 60:340-8.

20. Engel J, Eckel R, Schubert-Fritschle G, et al. Moderate progress for ovarian cancer in the last 20 years: Prolongation of survival, but no improvement in the cure rate. Eur J Cancer 2002; 38:2435-45.

21. Jain S, Shukla RC. MRI is a state of art imaging modality in characterization of indeterminate adnexal masses. Journal of Biomedical Science and Engineering. 2013; 6:29466.

22. S Radhamani1, M V Akhila2, Evaluation of adnexal masses - correlation of clinical, sonological and histopathological findings in adnexal masses, International Journal of Scientific Study, 2017; 4:11.

23. Iyer VR, Lee SI. MRI, CT, and PET/CT for ovarian cancer detection and adnexal lesion characterization. AJR Am J Roentgenol. 2010; 194(2):311-21. doi: 10.2214/ AJR.09.3522.

24. Li W, Zhang Y, Cui Y, Zhang P, Wu X. Pelvic inflammatory disease: Evaluation of diagnostic accuracy with conventional MR with added diffusion-weighted imaging. Abdomlmaging. 2013; 38(1):193-200.

\begin{tabular}{|c|c|c|c|}
\hline \multicolumn{4}{|c|}{ AUTHORSHIP AND CONTRIBUTION DECLARATION } \\
\hline Sr. \# & Author(s) Full Name & Contribution to the paper & Author(s) Signature \\
\hline 1 & Syed Anjum Mehdi & & \\
\hline 2 & Hassan Bukhari & & \\
\hline 3 & Shama Aslam & Equal Contribution all author & \\
\hline 4 & M. Sohail Anjum Noor & & \\
\hline
\end{tabular}

